\title{
Characterization of Surfaces and the Estimation of Shock Induced Vorticity
}

\author{
L. Jameson, J. Ray, and T. Peyser
}

September 17, 2002 


\section{DISCLAIMER}

This document was prepared as an account of work sponsored by an agency of the United States Government. Neither the United States Government nor the University of California nor any of their employees, makes any warranty, express or implied, or assumes any legal liability or responsibility for the accuracy, completeness, or usefulness of any information, apparatus, product, or process disclosed, or represents that its use would not infringe privately owned rights. Reference herein to any specific commercial product, process, or service by trade name, trademark, manufacturer, or otherwise, does not necessarily constitute or imply its endorsement, recommendation, or favoring by the United States Government or the University of California. The views and opinions of authors expressed herein do not necessarily state or reflect those of the United States Government or the University of California, and shall not be used for advertising or product endorsement purposes.

This work was performed under the auspices of the U. S. Department of Energy by the University of California, Lawrence Livermore National Laboratory under Contract No. W-7405-Eng-48.

This report has been reproduced directly from the best available copy.

Available electronically at http://www.doc.gov/bridge

Available for a processing fee to U.S. Department of Energy

And its contractors in paper from

U.S. Department of Energy

Office of Scientific and Technical Information

P.O. Box 62

Oak Ridge, TN 37831-0062

Telephone: (865) 576-8401

Facsimile: (865) 576-5728

E-mail: reports@adonis.osti.gov

Available for the sale to the public from

U.S. Department of Commerce

National Technical Information Service

5285 Port Royal Road

Springfield, VA 22161

Telephone: (800) 553-6847

Facsimile: (703) 605-6900

E-mail: orders@ntis.fedworld.gov

Online ordering: http://www.ntis.gov/ordering.htm

OR

Lawrence Livermore National Laboratory

Technical Information Department's Digital Library

http://www.llnl.gov/tid/Library.html 


\title{
Characterization of Surfaces and the Estimation of
}

\section{Shock Induced Vorticity}

\author{
L. Jameson, J. Ray ${ }^{1}$ and T. Peyser
}

Lawrence Livermore National Laboratory

University of California

P.O. Box 808, MS L-039

Livermore, CA 94551

Email: \{jameson3, peyser1\}@llnl.gov

\section{Abstract}

When shocks impinge on a surface separating fluids of two different densities, one observes the development and growth of various vortical structures. The flow induced by this Richtmyer-Meshkov (RM) instability depends on a variety of factors such as the shock strength, the density ratio of the fluids and the exact form of the surface perturbation. The most common way to model the form of the surface perturbation is through Fourier analysis which is suitable for large-scale sinusoidal structures and is straightforward mathematically. In surfaces of practical interest, however, to a wide range of application, there may also be a broad spectrum of high frequency modes in addition to the lower frequency modes described by Fourier methods. We propose here that these high frequency modes can be efficiently quantified in terms of wavelet analysis.

From a numerical point of view, the scale that the roughness occurs at is far to small to numerically resolve and thus we propose that our new methodology can be used to model the subgrid scale generation of vorticity. Thus the combination of wavelet analysis and Fourier analysis will be used to model the generation of vorticity for the RM instability.

'MS 9056, Sandia National Laboratories, Livermore CA 94551-0969; jairay@ca.sandia.gov 


\section{Introduction}

Material surfaces when examined on a fine scale will appear quite rough due to imperfections in the finishing process. Such fine scale information is far below resolving capabilities of modern numerical simulations. When shocks interact with density-stratified interfaces (the Richtmyer-Meshkov Instability,[1], [2]) they deposit baroclinic vorticity at the interface. The deposition, and the interfaces' subsequent evolution is affected by the large and small scales in equal measure. In case the fine scales cannot be resolved in a numerical simulation, gaining an understanding of the vorticity dynamics and mixing at the interface requires one to develop a subgrid scale model for this vorticity generation. We propose here a method for modeling the subgrid scale information at the material interface based on wavelet analysis and combine this new method with the traditional methodology of modeling surfaces that based on Fourier analysis or spherical harmonics.

Wavelet Analysis provides a new orthogonal basis set which is localized in both the physical space and the Fourier transform space. This localization makes wavelets an effective basis for the representation of localized features. Fourier analysis, on the other hand, provides an effective basis set in which to represent global, smooth, and periodic features. Many physical systems, however, are comprised of global smooth structure with localized small features superimposed. It is thus natural to represent such surfaces by combining a global smooth basis such as a Fourier or spherical harmonic basis with a local basis such as wavelet analysis. When shocks impinge on a surface modeled in this manner, a mixing region will immediately be induced from the portion of the representation modeled by wavelet analysis. This mixing region will also grow and be displaced with time. The quantification of mix in an analysis with a wavelet-resolved/ modeled subgrid structures will be drastically different from one where these have been ignored to obtain a clean bubble-spike configuration. We take the first step in that direction by quantifying the vorticity deposition on the surface due to each of these bases.

\section{Generation of Vorticity}

Circulation deposition is the dominant fluid dynamical process in early time Richtmyer-Meshkov instability environment; its quantification provides a measure of the interfacial mixing as well as a basis for model building and scaling. The physical problem may be characterized by a shock wave of Mach Number $M$ propagating in a rectangular shock-tube through a gas of density $\rho_{0}$ (and ratio of specific heats $\gamma_{0}$ ), and an interface separating a gas of density $\rho_{b}$ (and ratio of specific heats $\gamma_{b}$ ). Both the gases are assumed to be stationary. The parameter space is five dimensional : the shock strength (characterized by $\xi(M)=$ 
$\left(p_{1}-p_{0}\right) / p_{1}$, the normalized pressure jump across the incident shock), the stratification (characterized by $\left.\eta=\rho_{b} / \rho_{0}\right)$, the geometry of the interface, $\gamma_{b}$ and $\gamma_{0}$. Note that $\eta$ is related to the better known Atwood number by

$$
\text { At }=\frac{\eta-1}{\eta+1}
$$

The incident shock refracts at the interface and bifurcates into a transmitted shock and a reflected wave.

There are two generic classes of interaction : "slow-fast" ( $\mathrm{s} / \mathrm{f})$ and "fast/slow" (f/s). If the speed of the transmitted shock is higher (lower) than the incident shock, then the interaction is termed slow-fast (fastslow). For the parameter regime considered in this paper, it is sufficient to examine the sound speed in the two media to determine whether the interaction is $s / f$ or $f / s$. We will focus on $f / s$ interactions where the density ratio $\eta$ is greater than unity and where the reflected wave is a shock wave, which is typical for a fast-slow refraction. If the local angle between the shock and the interface is smaller than a critical angle $\alpha(M, \eta)$, the incident, reflected and transmitted shocks all meet at a node on the interface and the refraction is termed regular.

The vorticity evolution equation in $3 \mathrm{D}$ inviscid compressible flow is

$$
\frac{D \omega}{D t}=\frac{\nabla \rho \times \nabla p}{\rho^{2}}+\omega \cdot \nabla \mathbf{u}-\omega \nabla \cdot \mathbf{u}
$$

The derivation of Eq. (1) can be found in [5]. The first term on the right in Eq. (1) is the baroclinic source term. As the shock traverses the interface, a misalignment of pressure and density gradients leads to rapid vorticity deposition on the interface. After the rapid vorticity deposition phase, the slower evolution of the interface is driven mainly by the baroclinic vorticity deposited earlier. In this study the gas interface is initially vorticity free. Hawley and Zabusky [6] proposed a vortex paradigm to explain the evolution of a sawtooth perturbation in terms of vortex dynamics. Sturtevant [7] investigated interfaces with large sawtooth perturbations where he observed the formation of a large stratified wall vortex. This was corroborated by Yang, Chern et. al. [8] who employed Hawley and Zabusky's vortex paradigm to explain the evolution of the shocked interface

Samtaney and Zabusky [9] developed exact and approximate expressions for circulation deposition for fast/slow ( $f / s$ ) interfaces using shock polar analysis for simple interfaces (oblique, sinusoidal and circular). Their approach was extended to $s / f$ interfaces with regular shock refractions (refractions with a transmitted shock and a reflected centered expansion (RRE)) in [10].

In this study, we will attempt to develop an estimation of the vorticity deposition on "generalized" $2 \mathrm{D}$ surfaces. We assume that the surface is described by the superposition of two types of basis functions. The smooth, global and/or periodic charateristics of the surface will be captured with a harmonic function 
(Fourier analysis on a line or plane or spherical harmonics on the surface of a sphere) while the sharp, localized features will be represented using wavelets. Wavelets are very localized and not very smooth and are thus suitable for representing and analyzing data that is of the same localized "rough" nature. These two disparate basis sets can be made to work together in order to get an efficient representation of information that contains both global smooth perturbations and local rough perturbations that are superimposed.

Our analysis will, therefore, proceed assuming that the interface between the two density fields is represented by a superposition of harmonic functions and wavelets. Since wavelets are less well-known than harmonic basis function, we will begin our analysis assuming only a wavelet representation for the interface,

$$
I(y)=\sum_{j, k} d_{j, k} \psi_{j, k}(y)
$$

for a one dimensional interface manifold in a two dimensional experiment and,

$$
I(x, y)=\sum_{j_{1}, j_{2}, k_{1}, k_{2}} d_{j_{1}, j_{2}, k_{1}, k_{2}} \psi_{j_{1}, j_{2}, k_{1}, k_{2}}(x, y)
$$

for the two dimensional interface manifold in the three dimensional experiment. We will assume that the vertical shock wave impinges orthogonally on the surface in both two and three dimensions. A 2D example of a shock impinging on an interface is seen in Fig. 4(a).

In our next section (Section 3 ) we introduce wavelet analysis and the notation that we will need throughout the remainder of the manuscript. An extension of this wavelet analysis to $3 \mathrm{D}$ with spherical harmonics in Appendix B. In Section 4 we will derive scaling expressions (in $M$ and $\eta$ ) for the circulation deposition on a sinusoidal interface with superimposed Haar wavelets. In Section 5 we will present numerical solutions of the Euler equations, simulating shock interactions with sinusoidal interfaces with a set of identical Haars wavelets (but translated along the lattice of wavelet basis functions) and compare the scaling expressions vis-a-vis the numerical simulations.

\section{Wavelet Analysis}

Possibly the most instructive way to think of wavelets is in contrast to traditional analysis techniques such as Fourier analysis. With Fourier analysis we analyze discrete or continuous data using basis functions which are global, smooth and periodic. This analysis yields a set of coefficients, say, $a_{k}$, which gives the amount of energy in the data at frequency $k$. Wavelet analysis, by contrast, analyzes data with basis functions which are local, slightly smooth, not periodic, and which vary with respect to scale and location. Wavelet analysis thereby produces a set of coefficients $b_{j, k}$ which give the amount of energy in the data at scale $j$ 
and location $k$. Wavelet analysis can serve as a good complement to Fourier analysis. In fict. data which is efficiently analyzed with Fourier analysis often is not efficiently analyzed with wavelet analysis and the opposite situation also holds.

For our purposes here we will confine our discussion to the so-called orthogonal wavelets and specifically the Daubechies family of wavelets. The orthogonality property leads to a clear indicalion when data deviates from a low-order polynomial, the importance of which will become clear when we discuss numerical methods.

\subsection{Theoretical Background in the Continuous World}

To define Daubechies-based wavelets, see Daubechies (1988) and Erlebacher (1996), consider the two functions $\phi(x)$, the scaling function, and $\psi(x)$, the wavelet. The scaling function is the solution of the dilation equation,

$$
\phi(x)=\sqrt{2} \sum_{k=0}^{L-1} h_{k} \phi(2 x-k),
$$

which carries the name "dilation equation" since the the independent variable $x$ appears alone on the left hand side but is multiplied by 2 , or dilated, on the right hand side. One also requires the the scaling function $\phi(x)$ be normalized: $\int_{-\infty}^{\infty} \phi(x) d x=1$. The wavelet $\psi(x)$ is defined in terms of the scaling function,

$$
\psi(x)=\sqrt{2} \sum_{k=0}^{L-1} g_{k} \phi(2 x-k) .
$$

One builds an orthonormal basis from $\phi(x)$ and $\psi(x)$ by dilating and translating to get the following functions:

$$
\phi_{k}^{j}(x)=2^{-\frac{i}{2}} \phi\left(2^{-j} x-k\right)
$$

and

$$
\psi_{k}^{j}(x)=2^{-\frac{i}{2}} \psi\left(2^{-j} x-k\right)
$$

where $j, k \in Z$. $j$ is the dilation parameter and $k$ is the translation parameter.

It is usual to let the spaces spanned by $\phi_{k}^{j}(x)$ and $\psi_{k}^{j}(x)$ over the parameter $k$, with $j$ fixed, be denoted by $V_{j}$ and $W_{j}$ respectively,

$$
\begin{gathered}
V_{j}={ }_{k \in Z}^{\operatorname{span}} \phi_{k}^{j}(x), \\
W_{j}={ }_{k \in Z}^{\operatorname{span}} \psi_{k}^{j}(x) .
\end{gathered}
$$


The spaces $V_{j}$ and $W_{j}$ are related by,

$$
\cdots \subset V_{1} \subset V_{0} \subset V_{-1} \subset \ldots
$$

and

$$
V_{j}=V_{j+1} \bigoplus W_{j+1}
$$

where the notation $V_{0}=V_{1} \oplus W_{1}$ indicates that the vectors in $V_{1}$ are orthogonal to the vectors in $W_{1}$ and the space $V_{0}$ is simply decomposed into these two component subspaces.

The coefficients $H=\left\{h_{k}\right\}_{k=0}^{L-1}$ and $G=\left\{g_{k}\right\}_{k=0}^{L-1}$ are related by $g_{k}=(-1)^{k} h_{L-k}$ for $k=0, \ldots, L-1$. All wavelet properties are specified through the parameters $H$ and $G$. If one's data is defined on a continuous domain such as $f(x)$ where $x \in R$ is a real number then one uses $\phi_{k}^{j}(x)$ and $\psi_{k}^{j}(x)$ to perform the wavelet analysis. If, on the other hand, one's data is defined on a discrete domain such as $f(i)$ where $i \in Z$ is an integer then the data is analyzed, or filtered, with the coefficients $H$ and $G$. In either case, the scaling function $\phi(x)$ and its defining coefficients $H$ detect localized low frequency information, i.e., they are lowpass filters (LPF), and the wavelet $\psi(x)$ and its defining coefficients $G$ detect localized high frequency information, i.e., they are high-pass filters (HPF). Specifically, $H$ and $G$ are chosen so that dilations and translations of the wavelet, $\psi_{k}^{j}(x)$, form an orthonormal basis of $L^{2}(R)$ and so that $\psi(x)$ has $M$ vanishing moments which determines the accuracy. In other words, $\psi_{k}^{j}(x)$ will satisfy

$$
\delta_{k l} \delta_{j m}=\int_{-\infty}^{\infty} \psi_{k}^{j}(x) \psi_{l}^{m}(x) d x
$$

where $\delta_{k l}$ is the Kronecker delta function, and the accuracy is specified by requiring that $\psi(x)=\psi_{0}^{0}(x)$ satisfy

$$
\int_{-\infty}^{\infty} \psi(x) x^{m} d x=0
$$

for $m=0, \ldots, M-1$. This statement on accuracy can be explained in an alternative manner. Recall that within the scaling function subspaces $V_{j}$ one can reconstruct low order polynomials exactly up to a given order so that $x^{0}, x^{1}, \ldots, x^{M-1}$ can be represented exactly by appropriately choosing scaling function coefficients. Accordingly, the subspace $W_{j}$ which is orthogonal to $V_{j}$ and consequently the basis elements $\psi_{j}(x)$ of $W_{j}$ will be orthogonal to all elements contained in $V_{j}$ such as these low-order polynomials. So, one can see that by specifying the number of vanishing moments of the wavelets one has in effect specified the number of polynomials which can be represented exactly and hence the numerical accuracy of the method.

For representing functions in $L^{2}(R)$ one can see from the above expressions that for any function $f(x) \in$ $L^{2}(R)$ there exists a set $\left\{d_{j k}\right\}$ such that

$$
f(x)=\sum_{j \in Z} \sum_{k \in Z} d_{k}^{j} \psi_{k}^{j}(x)
$$


where

$$
d_{k}^{j}=\int_{-\infty}^{\infty} f(x) \psi_{k}^{j}(x) d x
$$

The two sets of coefficients $H$ and $G$ are known as quadrature mirror filters. For Daubechies wavelets the number of coefficients in $H$ and $G$, or the length of the filters $H$ and $G$, denoted by $L$, is related to the number of vanishing moments $M$ by $2 M=L$. For example, the famous Haar wavelet is found by defining $H$ as $h_{0}=h_{1}=1$. For this filter, $H$, the solution to the dilation equation (4), $\phi(x)$, is the box function: $\phi(x)=1$ for $x \in[0,1]$ and $\phi(x)=0$ otherwise. The Haar function is very useful as a learning tool, but because of its low order of approximation accuracy and lack of differentiability it is of limited use as a basis set. The coefficients $H$ needed to define compactly supported wavelets with a higher degree of regularity can be found in Daubechies (1988). As is expected, the regularity increases with the support of the wavelet. The usual notation to denote a Daubechies-based wavelet defined by coefficients $H$ of length $L$ is $D_{L}$.

\subsection{Practical Implementation in the Discrete World}

Naturally, infinite sums and integrals are meaningless when one begins to implement a wavelet expansion on a computer. One must find appropriate ways to implement discrete counterparts to the continuous operations which were outlined in the previous subsection. That is, nothing is continuous on a computer and since the original wavelet analysis was derived in the continuous world of differential and integral mathematics is it necessary to discuss a bit concerning a discrete version of the above continuous theory. Generally, operations such as integration are easily approximated with an appropriate order quadrature formula, but one would like to use as few quadratures as possible to limit the number of approximations which are made. We will see in this section how we can easily perform all the wavelet decompositions with relatively few approximations.

In a continuous wavelet expansion functions with arbitrarily small-scale structures can be represented. In practice, however, there is a limit to how small the smallest structure can be depending on, e.g., the numerical grid resolution or the sampling frequency in a signal processing scenario. Hence, on a computer an approximation would be constructed in a finite space such as

$$
V_{0}=W_{1} \oplus W_{2} \oplus \ldots \oplus W_{J} \oplus V_{J}
$$

with the approximation being

$$
P_{V_{0}} f(x)=\sum_{k \in Z} s_{k}^{J} \phi_{k}^{J}(x)+\sum_{j=1}^{J} \sum_{k \in Z} d_{k}^{j} \psi_{k}^{j}(x),
$$

with

$$
d_{k}^{j}=\int_{-\infty}^{\infty} f(x) \psi_{k}^{j}(x) d x
$$




$$
s_{k}^{J}=\int_{-\infty}^{\infty} f(x) \phi_{k}^{J}(x) d x
$$

utilizing orthogonality. Within this expansion, the scale $j=0$ is arbitrarily chosen as the finest scale required, and scale $J$ would be the scale at which a kind of local average, $\phi_{k}^{J}(x)$, provides sufficient large scale information, i.e. the first term in Eq.(16) provides the local mean around which the function oscillates.

One must also limit the range of the location parameter, $k$ Assuming periodicity of $f(x)$ implies periodicity on all wavelet coefficients, $s_{k}^{j}$ and $d_{k}^{j}$, with respect to $k$. For the non-periodic case, since $k$ is directly related to the location, a limit is imposed on the values of $k$ when the location being addressed extends beyond the boundaries of the domain.

The wavelet decomposition matrix is the matrix embodiment of the dilation equation, Eq.(4), defining the scaling function and the accompanying equation defining the wavelet, Eq.(5). The following two recurrence relations for the coefficients, $s_{k}^{j}$ and $d_{k}^{j}$, in Eq.(16) are given as

$$
s_{k}^{j}=\sum_{n=1}^{L} h_{n} s_{n+2 k-2}^{j-1}, d_{k}^{j}=\sum_{n=1}^{L} g_{n} s_{n+2 k-2}^{j-1},
$$

as obtained from Eqs.(4)-(5), and we recall that $h_{n}$ refers to the chosen filter while we have $g_{n}=-(-1)^{n} h_{L-n}$

Denote the decomposition matrix embodied by these two equations, assuming periodicity, by $P_{N}^{j, j+1}$ where the matrix subscript denotes the size of the square matrix while the superscripts indicate that $P$ is decomposing from scaling function coefficients at scale $j$ to scaling function and wavelet function coefficients at scale $j+1$, i.e. $P_{N}^{j, j+1}$ maps $\vec{s}_{j}$ onto $\vec{s}_{j+1}$ and $\vec{d}_{j+1}$ :

$$
P_{N}^{j, j+1}:\left[\vec{s}_{j}\right] \rightarrow\left[\begin{array}{c}
\vec{s}_{j+1} \\
\vec{d}_{j+1}
\end{array}\right],
$$

where we by $\vec{s}_{j}$ refer to the vector containing the coefficients at scale $j$. Note that the vectors at scale $j+1$ are half as long as the vectors as scale $j$.

Suppose, for illustration, the wavelet being used is the four coefficient $D_{4}$ wavelet, and that one wants to project from 8 scaling function coefficients at scale $j$ to 4 scaling function coefficients at scale $j+1$ and 4 wavelet coefficients at scale $j+1$. The decomposition matrix for the case of periodic boundary conditions, 
$P_{8}^{j, j+1}$, thus becomes

$$
P_{8}^{j, j+1} \equiv\left[\begin{array}{cccccccc}
h_{1} & h_{2} & h_{3} & h_{4} & 0 & 0 & 0 & 0 \\
0 & 0 & h_{1} & h_{2} & h_{3} & h_{4} & 0 & 0 \\
0 & 0 & 0 & 0 & h_{1} & h_{2} & h_{3} & h_{4} \\
h_{3} & h_{4} & 0 & 0 & 0 & 0 & h_{1} & h_{2} \\
g_{1} & g_{2} & g_{3} & g_{4} & 0 & 0 & 0 & 0 \\
0 & 0 & g_{1} & g_{2} & g_{3} & g_{4} & 0 & 0 \\
0 & 0 & 0 & 0 & g_{1} & g_{2} & g_{3} & g_{4} \\
g_{3} & g_{4} & 0 & 0 & 0 & 0 & g_{1} & g_{2}
\end{array}\right]
$$

where the periodicity is reflected in the coefficients being wrapped around. If the boundary conditions are not periodic, then one must extend the data and the scaling function coefficients at every scale in a "smooth" manner. This involves extending the scaling function data with a polynomial one order higher than the number of vanishing moments of the wavelet. But, for the purpose of understanding the method, it is sufficient to study the periodic matrix such as the one that we have created above. It is a matrix such as $P^{j, j+1}$ that is applied directly to the data and then directly to each level of scaling function coefficients. To be perfectly correct one would first approximate the scaling function coefficients at the finest scale using the raw data, however, in practice it seems to make very little difference if one simply considers the raw data to be the scaling function coefficients. So, for our purposes here we will simply use the raw data as the scaling function coefficients on the finest scale. The repeated application of the matrix $P^{j, j+1}$ yields the wavelet coefficients at the various scales, and it is these wavelet coefficients that provide a guide to the errors committed during the numerical calculation. Let us consider that the raw data is given and it is assumed to be the scaling function coefficients on the finest scale, $\vec{s}_{0}$. One wavelet decomposition yields the scaling function coefficients and wavelet coefficients at scale $j=1, \vec{s}_{1}$ and $\vec{d}_{1}$. A second application of the wavelet decomposition matrix will yield the vectors $\vec{s}_{2}$ and $\vec{d}_{2}$. It is the vectors $\vec{d}_{1}, \vec{d}_{2}, \ldots$ which yield the critical information on the numerical errors. If, for example, one sees that the values of the $\vec{d}_{1}$ are relatively large in the middle of the vector, then it is clear that within this one dimensional vector the largest errors will be in the middle of the one dimensional domain from which this vector was derived.

\subsection{Wavelet Analysis in 2D}

Generally, our fields of interest will be in two or three dimensions so must make a note on higher dimensional wavelet transforms. The approach that is most commonly taken is to use the tensor product of the one dimensional transform in order to generate the two dimensional transform. Recall that in one dimension that 
we can break up the subspace $V_{0}$ such that,

$$
V_{0}=V_{1} \oplus W_{1}
$$

So, if one takes the tensor product of this one dimensional analysis with another one dimensional analysis then one obtains,

$$
V_{0} \otimes V_{0}=\left(V_{1} \oplus W_{1}\right) \otimes\left(V_{1} \oplus W_{1}\right)
$$

which yields

$$
V_{0} \otimes V_{0}=\left(V_{1} \otimes V_{1}\right) \oplus\left(V_{1} \otimes W_{1}\right) \oplus\left(W_{1} \otimes V_{1}\right) \oplus\left(W_{1} \otimes W_{1}\right)
$$

One can think of the subspace,

$$
V_{1} \otimes V_{1}
$$

as representing the horizontal and vertical average, low-pass filtering, of the information contained in $V_{0} \otimes$ $V_{0}$. The subspace

$$
V_{1} \otimes W_{1}
$$

represents a horizontal low-pass filtering process and a vertical high-pass filtering process. Such a subspace would capture horizontal edges. On the other hand, the subspace

$$
W_{1} \otimes V_{1}
$$

would represent a horizontal high-pass filtering process and a vertical low-pass filtering process. One would be able to detect vertical edges in such a subspace. Finally, when the high pass filter is applied in both the vertical and horizontal directions one arrives at the subspace,

$$
W_{1} \otimes W_{1}
$$

It is this subspace that we are primarily interested in since it will detect variation in both the horizontal and vertical directions. Note that as in the one dimensional case we certainly will perform many levels of wavelet decompositions, and the subspaces that we will primarily be interested in will be,

$$
W_{j} \otimes W_{j}
$$

for $j=1,2,3,4 \ldots$ Generally, decompositions up to $j=4$ will be sufficient. 


\section{Circulation deposition on interfaces}

Samtaney et al [9] developed an analytical expression for circulation by a shock of strength $M$ at a gas-gas interface with density ration $\eta, \eta>1$ deposition as

$$
\Gamma_{u n i l}=\sigma_{1}(M, \eta) \sin \alpha+\sigma_{3}(M, \eta) \sin ^{3} \alpha
$$

where $\Gamma_{u n i t}$ is the circulation deposition per unit original interface (scaled by $\sqrt{\gamma} / c_{0}$ ), $M$ is the Mach number of the shock impinging on an interface inclined at angle $\alpha$ and separating gases of density contrast $\eta$. $c_{0}$ is the speed of sound in the incident gas. In this section we derive a simpler, though less accurate model, which embodies the correct scaling in $M$ and $\eta$.

Fig. 1 shows an incident shock (Mach number $M$ ) propagating to the right. It traverses a "diffuse" interface (where the interface width $\delta_{i}$ is small compared to some other length scale e. g. the radius of curvature, or amplitude or wavelength of a sinusoidal interface or the distance over which the circulation deposition is being measured). The angle of the interface from the vertical is $\alpha$. Pressure and density in the unshocked transmitted gas are $P_{I}$ and $\rho_{I} . \rho_{T}$ is the density of the transmitted gas $\rho_{T} / \rho_{I}=\eta$

In reality, the shock, at the interface will refract into a transmitted and reflected wave, all of which will intersect at a node if $\alpha<\alpha_{c}$, a critical angle [9]. Also, the incident shock interacts with the diffuse interface and bends through it. We will ignore such shock structures $\approx O\left(\delta_{i}\right)$

Assumptions :

1. $\delta_{i} \ll L$, where $L$ is some length scale of the interface. This ensures that the interface is very sharp, but not discontinuous.

2. The density distribution in space is $G(x, y)$. So

$$
\rho=\rho_{T}+\left(\rho_{I}-\rho_{T}\right) G(x, y)
$$

For example,

$$
G(x, y)=\frac{1+\tanh \left(-\xi / \delta_{i}\right)}{2}
$$

where $\xi$ is the perpendicular distance of a point $(x, y)$ from the interface, as shown in Fig. 1 .

3. The pressure gradient $\nabla P$ is assumed to be horizontal i. e. perpendicular to the incident shock and the pressure jump is assumed to be equal to the pressure jump of the incident shock. In case of regular refraction, the incident, reflected and transmitted shocks meet at a node on the interface, which is where the circulation gets deposited. Picking the incident shock to decide the pressure gradient is 
approximate and is valid only when the reflected shock is weak i.e. when $\alpha$ is "small". However it does capture the effect of the strength of the incident shock wave.

Let the shock speed be $W=M c_{0}$ where $c_{0}$ is the speed of sound in the unshocked medium. Thus the pressure and density distribution in the incident gas at any time $t$ is given by

$$
\begin{aligned}
P & =P_{I}+\Delta P H\left(1-\frac{x}{W t}\right) \\
\rho & =\rho_{T}+\left(\rho_{I}-\rho_{T}\right) G
\end{aligned}
$$

where $H(\ldots)$ is the Heaviside function.

$$
\begin{aligned}
\nabla P & =\frac{\Delta P \delta(\beta)}{W t} \hat{\mathbf{i}} \\
\nabla \rho & =\left(\rho_{I}-\rho_{T}\right)\left[G_{x} \hat{\mathbf{i}}+G_{y} \hat{\mathbf{j}}\right]
\end{aligned}
$$

where $\beta=1-\frac{X_{s}}{W t}$, where $X_{s}$ is the shock position. Thus the baroclinic term is

$$
\frac{\nabla \rho \times \nabla P}{\rho^{2}}=-\frac{(\eta-1)}{\eta} \frac{\Delta P G_{y} \delta(\beta)}{W t \rho_{I} \eta\left[1-\frac{\eta-1}{\eta} G\right]^{2}} \hat{\mathbf{k}}
$$

Let $\Gamma$ be the circulation deposited by the shock on the interface in time $\tau$.

$$
\Gamma=-\int_{t=0}^{t=\tau} d t \int_{y=-\infty}^{y=\infty} \int_{x=-\infty}^{x=\infty} \frac{(\eta-1)}{\eta} \frac{\Delta P G_{y} \delta(\beta)}{W t \rho_{I} \eta\left[1-\frac{\eta-1}{\eta} G\right]} d x d y
$$

But

$$
\begin{aligned}
\beta=1-\frac{x}{W t} & \sim d \beta=-\frac{d x}{W t} \\
x=-\infty & \sim \beta=\infty \\
x=\infty & \sim \beta=-\infty
\end{aligned}
$$

So

$$
\begin{aligned}
\Gamma & =-\int_{t=0}^{t=\tau} d t \int_{y=-\infty}^{y=\infty} \int_{\beta=-\infty}^{\beta=\infty} \frac{(\eta-1)}{\eta} \frac{\Delta P}{\rho_{I} \eta} \frac{G_{y}}{\left[1-\frac{\eta-1}{\eta} G\right]^{2}} \delta(\beta) d \beta d y \\
& =\frac{\eta-1}{\eta} \frac{\Delta P}{\eta \rho_{I}} \int_{t=0}^{t=\tau} d t \int_{y=-\infty}^{y=\infty} \frac{G_{y} d y}{\left(1-\frac{\eta-1}{\eta} G\right)^{2}} \quad \text { for } \beta=0 \text { i. e. } x=W t \\
& =-\frac{\Delta P}{\rho_{I} \eta} \int_{t=0}^{t=\tau} d t \int_{Q=0}^{Q=\frac{\eta-1}{\eta}} \frac{d Q}{(1-Q)^{2}} \quad \text { where } \frac{\eta-1}{\eta} G=Q
\end{aligned}
$$




$$
\begin{aligned}
& =-\frac{\Delta P}{\rho_{I} \eta} \int_{t=0}^{l=\tau}(\eta-1) d t \\
& =-\frac{\Delta P(\eta-1) \tau}{\rho_{I} \eta} \\
& =-\frac{\Delta P}{\rho_{I}}\left(1-\frac{1}{\eta}\right) \frac{L \sin \alpha}{W}
\end{aligned}
$$

where $L \sin \alpha=X_{s}$ is the distance traveled along the $\mathrm{x}$-axis by the shock in time $\tau$. Note the integration limits on $Q$ were placed using the following relations:

$$
\begin{array}{ll}
y \rightarrow \infty \quad & \quad \xi \rightarrow-\infty, Q \rightarrow \frac{\eta-1}{\eta} \\
y \rightarrow-\infty \quad & \quad \xi \rightarrow \infty, Q \rightarrow 0
\end{array}
$$

Thus, the scaled circulation deposited per unit original interface length $\Gamma_{u n i t}\left(=\Gamma / L \times \sqrt{\gamma} / c_{0}\right)$ is

$$
\begin{aligned}
\Gamma_{u n i t}=\frac{\Gamma}{L} \frac{\sqrt{\gamma}}{c_{0}} & =-\frac{2 \sqrt{\gamma}}{\gamma+1}\left(1-\frac{1}{\eta}\right)\left(M-\frac{1}{M}\right) \sin \alpha \\
& =\mu \sin \alpha
\end{aligned}
$$

where $\Delta P$, the pressure jump across the shock is replaced by $\frac{2 \gamma}{\gamma+1}\left(M^{2}-1\right)$ and $W=M c_{0}$. Simplifying

$$
\Gamma_{\text {unit }}=-\frac{2 \sqrt{\gamma}}{\gamma+1}\left(1+\frac{1}{\sqrt{\eta}}\right)\left(1-\frac{1}{\sqrt{\eta}}\right)\left(1+\frac{1}{M}\right)(M-1) \sin \alpha
$$

As $M \rightarrow 1$ and $\eta \rightarrow 1$, the expression approaches the one in [9].

Deduction 1

Given an arbitrary line $\mathcal{S}$ between points $A$ and $B$, the net circulation deposited is

$$
\Gamma=\int_{\mathcal{S}} \bar{\mu} \sin \alpha d s=\int_{\mathcal{S}} \bar{\mu} d x=\bar{\mu}\left(X_{B}-X_{A}\right)
$$

\subsection{Circulation deposition on a collection of Haar wavelets}

Thus far our analysis has been in a completely general setting allowing for any choice of wavelet. In practice, we expect that the Haar wavelet will be the wavelet of choice for surface characterization. The reason for this is that the Haar wavelet is the only wavelet in the Daubechies class of wavelets that does not overlap with the adjacent Haar wavelet that has been translated to the next position in the lattice of wavelet basis functions. Because of this non-overlap, we expect that the vorticity induced on adjacent and superimposed Haar wavelets will be additive. Thus, given an expression for the wavelet expansion of a surface, we expect 
to be able to simply add up the vorticity induced on each of the individual wavelet basis functions. We will now proceed to develop a model for the circulation deposited on an interface composed entirely of Haar wavelets.

Let there be a Haar-wavelet shaped interface as shown in Fig. 2, about to be crushed by a shock. Circulation is deposited on the sides of the Haar where $\sin \alpha \neq 0$ i.e on sides $\widehat{A B}, \widehat{C D}$ and $\widehat{E F}$. The circulations deposited are

$$
\begin{array}{ll}
\Gamma_{A B}=-\bar{\mu} H & y=0 \\
\Gamma_{C D}=2 \bar{\mu} H & y=S / 2 \\
\Gamma_{E F}=-\bar{\mu} H & y=S
\end{array}
$$

where $H$ is the height of the Haar wavelet and $S$ is the support. The net circulation on $A B \widehat{C D E F}$ is 0 as $X_{F}-X_{A}=0 . \bar{\mu}$ is the circulation deposition per unit interface length and can be $\sigma$ [9] or $\mu$ (as derived above) depending upon whether we want a scaling expression or an accurate measure of the circulation.

Define a wavelet $\psi_{j}(y)$ at scale $j$ as

$$
\begin{aligned}
\psi_{j}(y) & =2^{-j / 2} \psi_{0}\left(2^{-j} y\right) \\
& =2^{-j / 2}\left\{\phi\left(2^{-j} \cdot 2 y\right)-\phi\left(2^{-j} \cdot 2 y-1\right)\right\} \\
& =2^{-j / 2}\left\{\phi\left(\frac{2 y}{S_{j}}\right)-\phi\left(\frac{2 y}{S_{j}}-1\right)\right\}
\end{aligned}
$$

where $\psi_{0}(y)$ is the mother wavelet, $\phi$ its generating function and $S_{j}$ is the support of the wavelet at scale $j$. We define a $x$-integrated vorticity as

$$
\Omega(y)=\int_{-\infty}^{\infty} \omega_{i} d x
$$

where $\omega_{i}$ is the vorticity on the interface. Then, using $\sin \alpha= \pm 1$ or 0 depending upon the slope of the Haar, the $\Omega(y)$ for the Haar interface is

$$
\begin{aligned}
\Omega_{\psi_{j}} & =-2^{-j / 2} \bar{\mu}\left[\delta(y)-2 \delta\left(2^{-(j-1)} y-1\right)+\delta\left(2^{-(j-1)} y-2\right)\right] \\
& =-2^{-j / 2} \bar{\mu}\left[\delta\left(\frac{2 y}{S_{j}}\right)-2 \delta\left(\frac{2 y}{S_{j}}-1\right)+\delta\left(\frac{2 y}{S_{j}}-2\right)\right]
\end{aligned}
$$

and for a translated Haar

$$
\Omega_{\psi_{j, k}}=-2^{-j / 2} \bar{\mu}\left[\delta\left(\frac{2 y}{S_{j}}-k\right)-2 \delta\left(\frac{2 y}{S_{j}}-(k+1)\right)+\delta\left(\frac{2 y}{S_{j}}-(k+2)\right)\right]
$$


The circulation deposited on the interface $I(y)=\sum_{j, k} d_{j k} \psi_{j k}(y)$ is

$$
\Omega_{I(y)}=-\bar{\mu} \sum_{k} \sum_{j} d_{j k} 2^{-j / 2}\left[\delta\left(\frac{2 y}{S_{j}}-k\right)-2 \delta\left(\frac{2 y}{S_{j}}-(k+1)\right)+\delta\left(\frac{2 y}{S_{j}}-(k+2)\right)\right]
$$

We also define

$$
\begin{aligned}
& \Omega^{+}=\bar{\mu} \sum_{k} \sum_{j} d_{j k} 2^{-j / 2}\left[2 \delta\left(\frac{2 y}{S_{j}}-(k+1)\right)\right] \\
& \Omega^{-}=-\bar{\mu} \sum_{k} \sum_{j} d_{j k} 2^{-j / 2}\left[\delta\left(\frac{2 y}{S_{j}}-k\right)+\delta\left(\frac{2 y}{S_{j}}-(k+2)\right)\right]
\end{aligned}
$$

Note that these distributions change as the interface evolves in time; thus Eq. 37 is strictly valid only immediately after the shock-interface interaction.

Assuming the "horizontal" sides of the Haars at different scales and translations do not cancel each other, we can get the upper limits on the positive and negative circulations generated

$$
\begin{aligned}
\Gamma_{I}^{-}(y) & =\int_{0}^{L} \Omega^{-} d y=-\bar{\mu} \int_{0}^{L} d y \sum_{j k} d_{j k} \cdot 2^{-j / 2}\left[\delta\left(\frac{2 y}{S_{j}}-k\right)+\delta\left(\frac{2 y}{S_{j}}-(k+2)\right)\right] \\
& \geq-2 \bar{\mu} \sum_{j k}\left|d_{j k}\right| \cdot 2^{-j / 2} \\
\Gamma_{I}^{+}(y) & =\int_{0}^{L} \Omega^{+} d y=2 \bar{\mu} \int_{0}^{L} d y \sum_{j k} d_{j k} \cdot 2^{-j / 2}\left[\delta\left(\frac{2 y}{S_{j}}-(k+1)\right)\right] \\
& \leq 2 \bar{\mu} \sum_{j k}\left|d_{j k}\right| \cdot 2^{-j / 2}
\end{aligned}
$$

where $0 \leq y \leq L$

\section{Computational Examples}

In this section we compare Eq. 37 with numerical simulations to verify the suitability of $\Omega_{I(y)}$ as a scaling for the circulation on the interface. In order to simulate the interaction of a shock with a Haar-wavelet perturbed sinusoidal interface, we make the following assumptions : the flow is inviscid, the gases are perfect and there are no chemical reactions between the two gases, which are further assumed to be in thermal equilibrium. We have used the conservative level set formulation of Mulder et. al [11] to track the interface. The basic idea is as follows : Consider a function $\zeta(\mathbf{x}, t)$ which is defined everywhere in the domain. Then a particular 
value defines the interface. In our case, we initially use $\zeta(x, 0)=+1(-1)$ in the incident (transmitted) gas. We define the interface as $\zeta(\mathbf{x}, t)=0$. The function $\zeta(\mathbf{x}, t)$ is governed by the partial differential equation

$$
\partial_{\iota} \zeta+\mathbf{u} \cdot \nabla \zeta=0
$$

where $\mathbf{u}=\{u, v\}$ is the velocity vector

The governing equations (the compressible Euler equations) in conservative form are

$$
\mathbf{U}_{\iota}+\mathcal{F}(\mathbf{U})_{x}+\mathcal{G}(\mathbf{U})_{y}=0
$$

where

$$
\begin{aligned}
\mathbf{U} & =\{\rho, \rho u, \rho v, E, \rho \zeta\}^{T}, \\
\mathcal{F}(\mathbf{U}) & =\left\{\rho u, \rho u^{2}+p, \rho u v,(E+p) u, \rho \zeta u\right\}^{T}, \\
\mathcal{G}(\mathbf{U}) & =\left\{\rho v, \rho u v, \rho v^{2}+p,(E+p) v, \rho \zeta v\right\}^{T},
\end{aligned}
$$

$E$ is the total energy, related to the pressure $p$ by $p=(\gamma-1)\left(E-\frac{1}{2} \rho\left(u^{2}+v^{2}\right)\right)$ and $\zeta$ is the interface tracking function.

\subsection{Initial and boundary Conditions}

Fig. 4(a) shows a setup of the interaction being simulated. The boundary conditions are post-incident shock values at the left boundary and quiescent flow $\left(p_{b}, \rho_{b}, \mathbf{u}=0\right)$ at the right boundary. The upper and lower boundaries are perfectly reflecting i.e. $\mathbf{u} \cdot \mathbf{n}=0$, where $\mathbf{n}$ is the unit normal to the plane of the boundary. A sinusoidal interface with $A / \lambda=0.05$ separates the two gases - in the particular case, the density contrast is 10. The width of the shock tube was fixed at 1.0 , which corresponds to $\lambda / 2$ for the sinusoidal interface, $\lambda$ being the wavelength. Inscribed on the sinusoidal interface are Haars of scale $j=2$, i. e. $H_{j} / S_{j}=2^{-3 / 2 j}=$ $1 / 8$ where $H_{j}$ and $S_{j}$ are the height and support of the wavelet. Further, $S_{2} / \lambda=0.1 ;$ consequently, as seen in Fig. 4 , there are a little more than 5 wavelets on the interface. In the absence of the sine wave, the total interface length (per wavelet) on which circulation gets deposited is $4 \mathrm{H}_{2}$. Assuming that there are 5 wavelets on the interface, the total circulation-deposition area in the wavelets is $P_{j}$

$$
\frac{P_{j}}{\lambda}=5 \times \frac{4 H_{2}}{\lambda}=20 \times \frac{S_{2}}{8 \lambda}=\frac{20}{8} \times .1=\frac{1}{4}=5 \frac{A}{\lambda}
$$

The compressible Euler equations (with an extra equation for the interface tracker) were solved over a regular grid with A shock moving in the positive $\mathrm{x}$-direction is initialized using the Rankine-Hugoniot jump 
conditions. The shock was initialized at $X_{0}=10 \Delta x$ left of the interface which is spread over $2 \Delta x$ to $3 \Delta x$. The numerical method is described in Appendix $\mathrm{A}$

\subsection{Quantifications and Grid-Convergence}

We now quantify the circulation in the numerical simulations and compare it with the analytical results. At any instant the total interfacial vorticity is computed as

$$
\hat{\Gamma}(t)=\sum_{D} \omega(i, j, t) \Delta x \Delta y
$$

where $D=(i, j, t) \forall|\zeta(\mathrm{x}, t)|<1 . \omega(i, j, t)$ is computed using a second-order central difference as follows

$$
\omega(i, j, t)=\frac{v(i+1, j, t)-v(i-1, j, t)}{2 \Delta x}-\frac{u(i, j+1, t)-u(i, j-1, t)}{2 \Delta y} .
$$

We now need to decide the minimum resolution need to capture the vorticity deposition on a Haar wavelet. In the resolution convergence study we present the results of a Air-R22 interface (density contrast $3, \gamma_{0}=1.4, \gamma_{b}=1.1732$ ) accelerated by a Mach 1.5 shock. Half a Haar wavelet (scale $j=2$ i. e. $H_{2} / S_{2}=1 / 8$ ) is inscribed on a flat vertical interface. Note that only the large horizontal side of the Haar wavelet is included in the computational domain. Fig. 2 shows a schematic of the setup - however, since only half a Harr is considered, only $\widehat{C D}$ is present and the vertical sides extend to the shock-tube walls. In Fig. 3 we present the circulation deposited on the Haar wavelet when the "horizontal" part (equal to $2 \mathrm{H}_{2}$; where the vorticity is deposited) is resolved by $10 \Delta x, 20 \Delta x$ and $40 \Delta x$; these correspond to $S_{2}=40,80$ and $160 \Delta x$. The last two resolution do not change the circulation deposition much and we will henceforth, use $S_{2}=80 \Delta x$. As described in Section 5.1, $S_{2} / \lambda=0.1$ and so the sine wave will be resolved as $\lambda=800 \Delta x$.

\subsection{Description of the Shock - Interface Interaction}

In Fig. 4 we show the initial condition of a half a sinusoidal mode with Haar wavelets superimposed on it. A shock of Mach number $M=10$ propagates from the left. The density contrast $\eta=10$ and $\gamma_{0}=$ $\gamma_{b}=1.4$. We define $\tau$, the "interface-crushing" time of the shock, as the time needed by the interface to travel $2(A+H), A$ and $H$ being the amplitude of the sinusoidal perturbation and the height of the Haar respectively. In Fig. 4(b) to (f) we see the evolution of the flow at the times indicated in the caption. We see the shock crushing the interface, depositing positive and negative vorticity. In Fig. 4 (b) and (c) the Haar wavelets are seen to become mushroom shaped dipoles and move away from the original interface under self-induction. The corresponding times are $t / \tau=2.2$ and 5.99. By (d), we see that the dipoles widths are approximately $S_{j} . t / \tau=12.04$ for this frame. By (e) and (f), $(t / \tau=20.36,44.79)$ we see Haar-induced 
eddies comparable in size to the "wall vortex" [12] (bottom left in the last picture) and interact with each other strongly, similar to the bubble-competition described in [13]. Note that the portion of the interface represented by the wavelets, becomes turbulent very quickly and will comprise the mixing region that will be characterized by a mixing width. These, however, cannot be modeled properly using the (inviscid) Euler equation. The sinusoidal structure can be seen throughout the frames and in the later frames we identify the familiar bubble-spike structure of shock-sine wave interactions, though with a multiple roll-ups characterized by merged Haar-generated mushrooms. Thus we can identify three regimes here (a) an early time $t \sim O(\tau)$, when the baroclinic vorticity has been deposited but the wavelet evolution is still nascent (b) a regime where wavelet generated mushrooms become approximately $S_{j}$ in size and interact with their neighbors (Fig. 4(c) and (d)) and (c) late times when mushrooms merge to form a deformed bubble-spike interface.

In Figure (5) we plot the circulation for a Mach 1.5 shock and for $\eta=3.0$ (Air-R22, $\gamma_{0}=1.4, \gamma_{b}=$ 1.1732). In Figure (5) (below) we plot the circulation for a Mach 10 shock, $\eta=10$ and $\gamma_{0}=\gamma_{b}=1.4$. For each parameter set we plot the circulation on an interface with and without Haar wavelets. The net circulation is unaffected by the presence of Haars; as shown in Section 4, their net circulation is zero. Plotting the positive and negative circulation separately reveals the Haars' contributions; they have a little more circulation on them than the sinusoidal perturbation. Further, the circulation deposition in the $M=$ $10, \eta=10$ case is 20 times larger than the other.

The wavelet collection in Fig. 4(a) can be characterized as

$$
\Psi_{j}=\sum_{\kappa=0}^{4} \psi_{j(2 \kappa)}=H_{j} \sum_{\kappa=0}^{4}\left[\phi\left(\frac{2 y}{S_{j}}-2 \kappa\right)-\phi\left(\frac{2 y}{S_{j}}-2 \kappa-1\right)\right]
$$

where $H_{j}$ and $S_{j}$ are the height and the support of the Haar wavelets at scale $j$ respectively. Note that we have wavelets only of one scale, $j=2$. Also, Eq. 38 becomes

$$
\begin{aligned}
& \Omega^{-}=\bar{\mu} H_{j} \sum_{\kappa=0}^{4}\left[\delta\left(\frac{2 y}{S_{j}}-2 \kappa\right)+\delta\left(\frac{2 y}{S_{j}}-2 \kappa-2\right)\right] \\
& \Omega^{+}=\bar{\mu} H_{j} \sum_{\kappa=0}^{4}\left[-2 \delta\left(\frac{2 y}{S_{j}}-2 \kappa-1\right)\right]
\end{aligned}
$$

On the other hand, the sinusoidal perturbation on the interface, described as $x=-A \cos \pi y / L$ has a circulation distribution of

$$
\Omega_{\text {sine }}=\frac{2 \pi A \vec{\mu}}{\lambda} \sin \frac{2 \pi y}{\lambda}
$$


where $A$ is the amplitude and $L$ the width of the shock tube, $L=\lambda / 2$. Note that the circulation on the wavelets and the sinusoidal perturbation are

$$
\begin{aligned}
\Gamma_{\text {wavelet }}^{+} & =\int_{0}^{L} \Omega^{+} d y=\bar{\mu} \lambda / 8 \\
\Gamma_{\text {wavelet }}^{-} & =\int_{0}^{L} \Omega^{-} d y=-\bar{\mu} \lambda / 8 \\
\Gamma_{\text {sine }} & =\int_{0}^{L} \Omega_{\text {sine }} d y=2 \bar{\mu} A=\bar{\mu} \lambda / 10
\end{aligned}
$$

where the facts that $H_{j} / S_{j}=1 / 8, S_{j} / \lambda=1 / 10$ and $A / \lambda=0.05$ have been exploited. Thus the magnitude of the positive and negative circulation on the wavelets and that on the sinusoidal perturbation should be roughly the same, regardless of $M$ and $\eta$, as shown in Fig. 5. Note: Eq. 45 is valid immediately after circulation deposition and before interfacial evolution.

Eq. 37 can serve as a means of scaling the $\Omega$ distribution for a range of $M$ and density ratios $\eta$. $\Omega$ distributions change over time as the Haars and the sinusoidal interface evolves. Eq. 37 is valid only in the early time; as the Haars evolve and dipoles are formed (Fig. 4(c) and (d)), the "spiky" distribution predicted by Eq. 37 will evolve into a saw-tooth configuration as shown in Fig. 6 and 7. We rewrite Eq. 37 for late times as $\Omega_{\psi_{j, k}}=Q \mathcal{S}_{S_{j}}(y)$ where $\mathcal{S}_{S_{j}}$ is the sawtooth function with wavelength $S_{j}$. Assuming that the circulation on a Haar remains the same (i.e. secondary baroclinic deposition by reflected shocks etc) is small, $Q=\bar{\mu}$. For scaling purposes we will use $\bar{\mu}=\mu$ rather than $\sigma$. Thus the scaling quantity for $\Omega$, $\Omega_{s}$, is $\Omega_{s}=\mu(M, \eta)\left(\mathcal{S}_{S_{j}}+W(y, t)\right)$, where $W$ is the time-evolved distribution of $\Omega$ on the sinusoidal

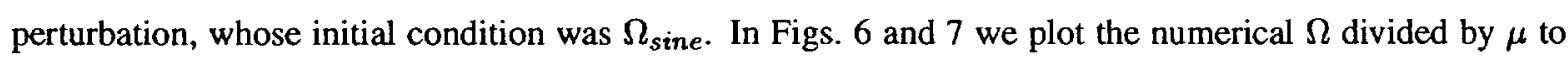
show the $(M, \eta)$ scaling. Even though the circulation deposition in the two cases differ by a factor of 20 (see Fig. 5), the scaling factor collapses them between 0.5 and 2.0. The initial "spiky" distribution of $\Omega_{I(y)}$ (Eq. 37) at $t / \tau \sim 1$ is seen to evolve into a smoother sawtooth distribution. As the base of the $\Omega$ distribution widens, the peaks fall, indicating a conservation of circulation in the Haar generated mushrooms. Later in time, when bubble-competition and merger occurs for these Haar-generated dipoles, one sees dual peaks / troughs within one $S_{j}$ region; this is particularly true for the $M=10, \eta=10$ run in Fig. 7 at $t / \tau=11.22$. We also note that even though the dipoles have grown to $S_{j}$ in width, and have translated away from the interface, they have not moved along it. Thus the peaks-and-troughs distribution in Figs. 6 and 7 at $t / \tau \sim 1$ are every similar to that at $t / \tau \sim 10$ 


\section{Conclusions}

Our goal in this manuscript has been to outline a new methodology that can be used to effectively measure the mix induced between two materials when a shock impinges on their interface. Traditionally such studies have been conducted by modeling the interface between the materials with Fourier analysis and resolving the modes in numerical simulations. Fine structures, inherent in any realistic material interface, have been typically ignored because of the large computational cost assocaited with resolving them. In this paper we have established Haar wavelets as a basis for represnting fine structures. A scaling model for the circulation deposition on such structures was developed and validated vis-a-vis Euler simulations. We find that though the Haar-generated mushrooms (dipoles) roll-up, widen and move away from the interface, they do not move along it. Thus the spatial circulation distribution, measured in an integrated-fashion along the interface remains largely unchanged till the widening mushrooms start interacting and merging with their neighbors. to form larger structures. At late times the familiar bubble-spike structure is recovered, but these are highly contorted by large vortical structures, originating from the merger of Haar-generated mushrooms. While this paper has captured the driving force (vorticity), the kinematics (the evolution of the Haars and their mergers) is left for future study. Our new method is intended to be a first step in overcoming the limitation of resolving small scale roughness on surfaces by modeling the subgrid scale vorticity generation with wavelet analysis and combining this information with the traditional Fourier approach. Thus far our analysis has been mainly on the theoretical level, but we intend in follow up work to draw closer the connection between the wavelet based subgrid scale model of vorticity generation to actual estimates of the physical mixing process between materials. 


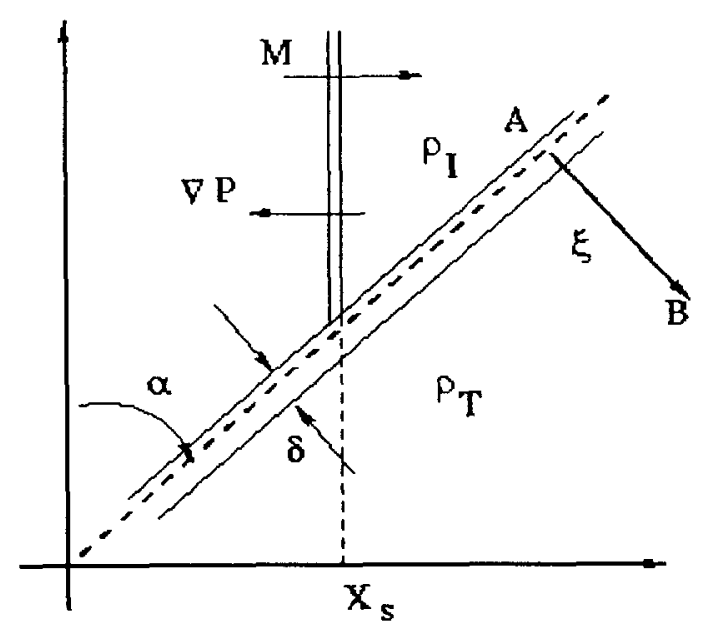

Figure 1: Schematic of a shock traversing an interface inclined at $\alpha$ to the shock. The incident gas of density $\rho_{I}$ is on the left; the transmitted gas $\rho_{T}$ on the right. The shock moves from left to right; its position on the $\mathrm{X}$-axis is $X_{s}$. The interface is diffused over a small width $\delta$.

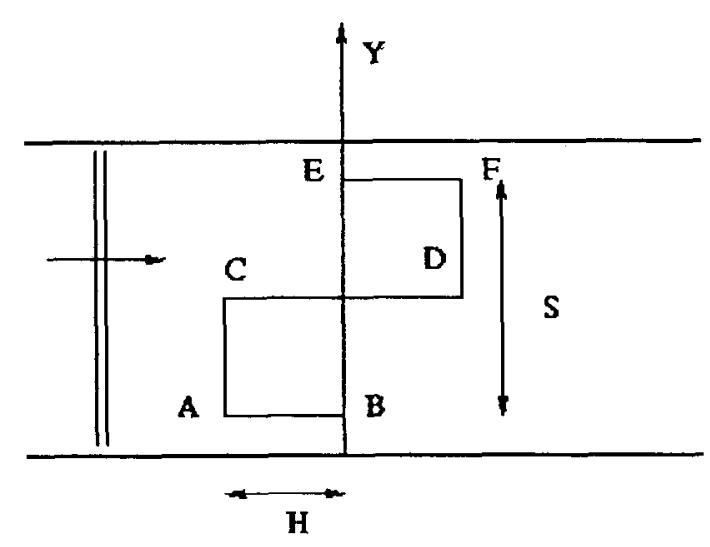

Figure 2: Schematic of a Haar wavelet on a vertical interface. The shock travels from the left to the right. Circulation is deposited only on $\widehat{A B}, \widehat{C D}$ and $\widehat{E F}$. The net circulation on the entire Haar wavelet is zero. $H$ and $S$ are the height and support of the Haar wavelet respectively. 


$$
L
$$



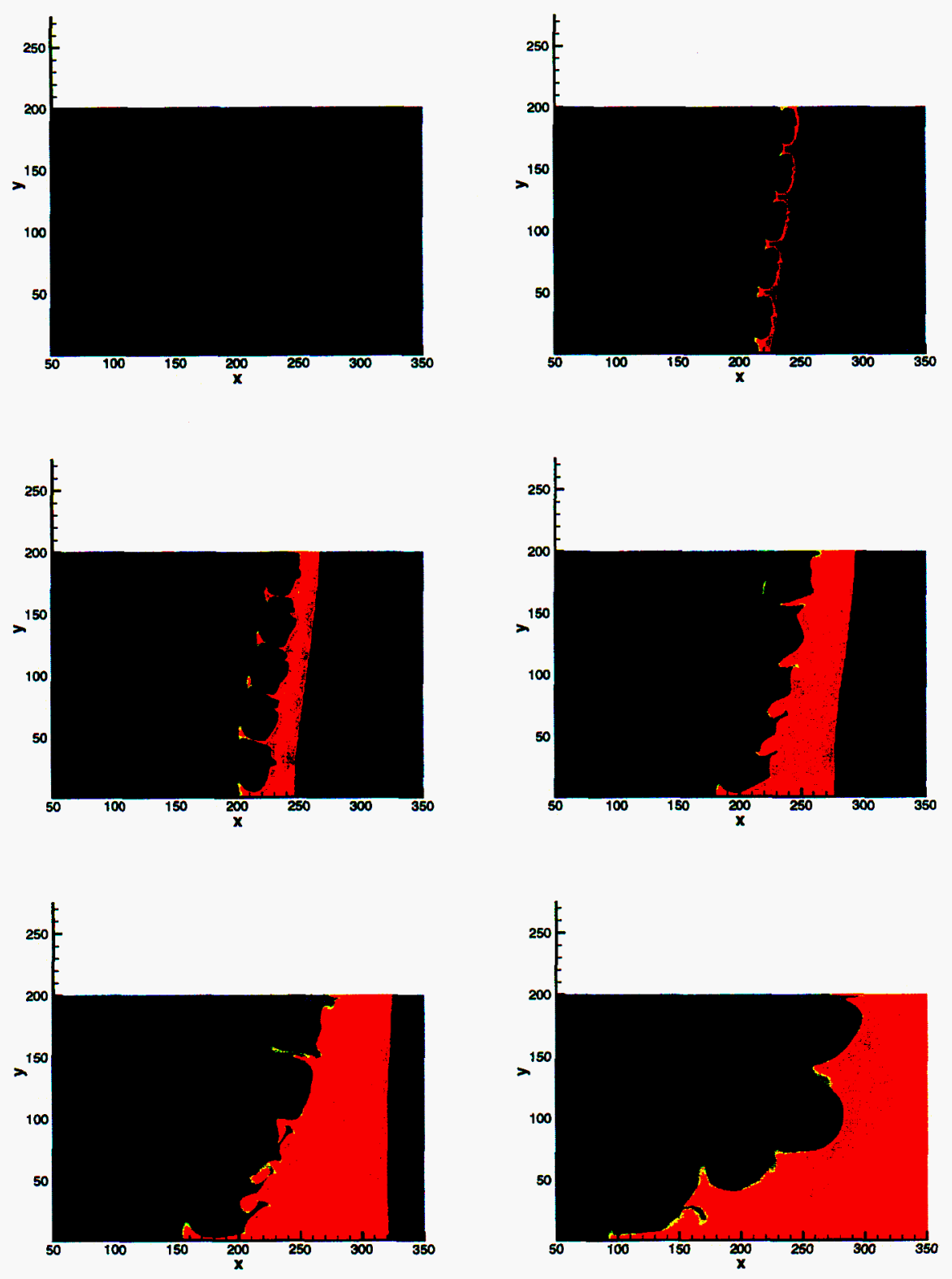

Figure 4: (a) - (f), counting left to right, top to bottom. Snapshots from a $M=10, \eta=10$ interaction with the interface described in Section 5.1. We plot the density map, overlaying the "interface-tracker" to denote the gas-gas interface. We see "jets", essentially vortex dipoles moving away from the sinusoidal interface. As time evolves, $(f)$, the interface shows the presence of eddies comparable in size to the wall vortex at the base of the shock tube, indicating a thickening of the interfacial region. The $x-$ and $y$ - axis units are in $\Delta x=\Delta y$. The time for the frames are $t / \tau=0.0,2.2,5.99,12.04,20.36$ and 44.79 respectively, where $\tau=2(A+H) / M c_{0}$ is the "interface-crushing" time of the shock. 

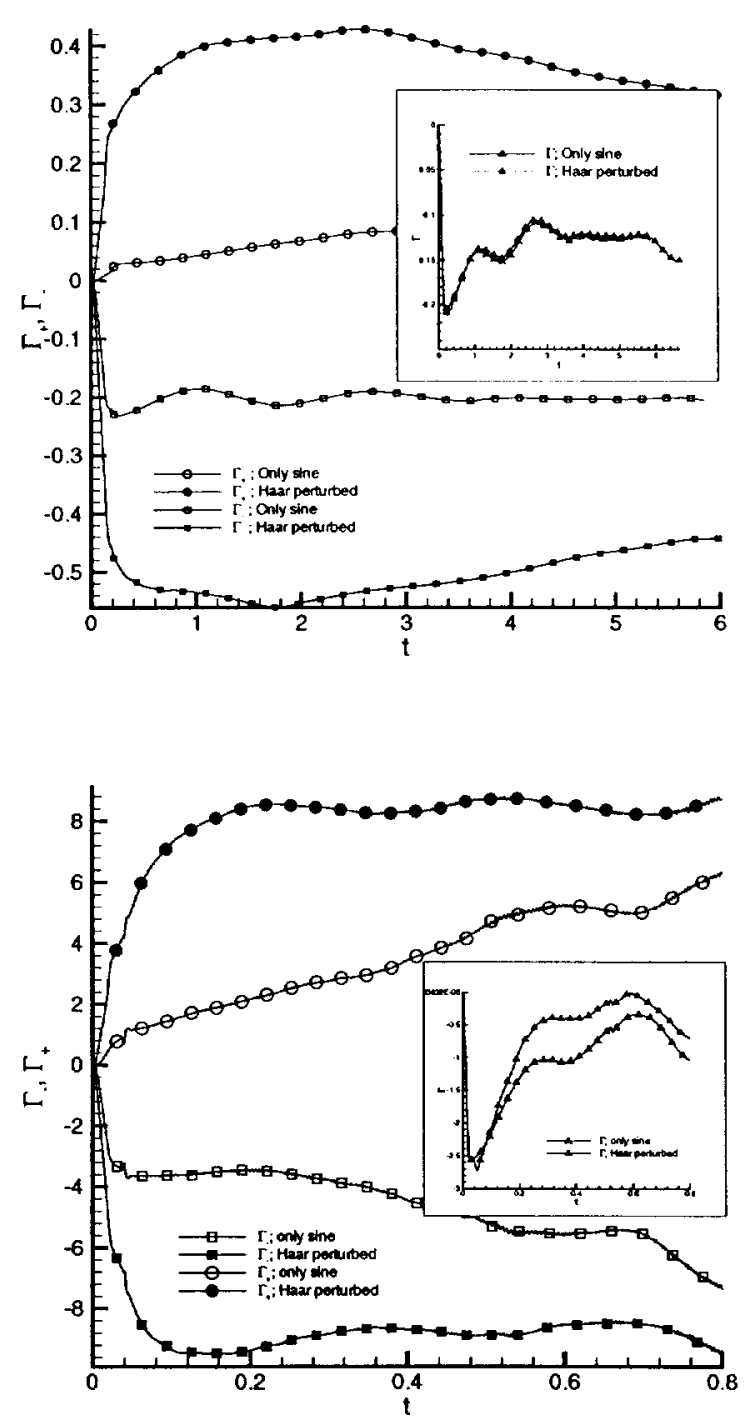

Figure 5: Above : Circulation for a $M=1.5$ shock interaction with $\eta=3$ interface as described in Section 5.3. We plot the net, positive $\left(\Gamma_{+}\right)$and negative circulation $\left(\Gamma_{-}\right)$on the interface for a sinusoidal interface with and without Haar perturbation. While the contribution of the Haars to the net circulation is zero, at least at early time, (Section 5.3; also Inset), their presence is felt in the positive and negative circulation budgets, which are almost double of the no-Haar values. Bottom : The observations are borne out for a $M=10$ shock with $\eta=10$ interface. The o symbol denotes positive circulation on the interface, $\square$ negative circulation and the $\triangle$ denotes the net circulation. Filled symbols are for Haar-perturbed interfaces. The interface crushing time (the time needed for the shock wave to travel a distance of $2(A+H)$ ) are 0.14 and 0.021 for the $M=1.5$ and 10.0 cases respectively. 


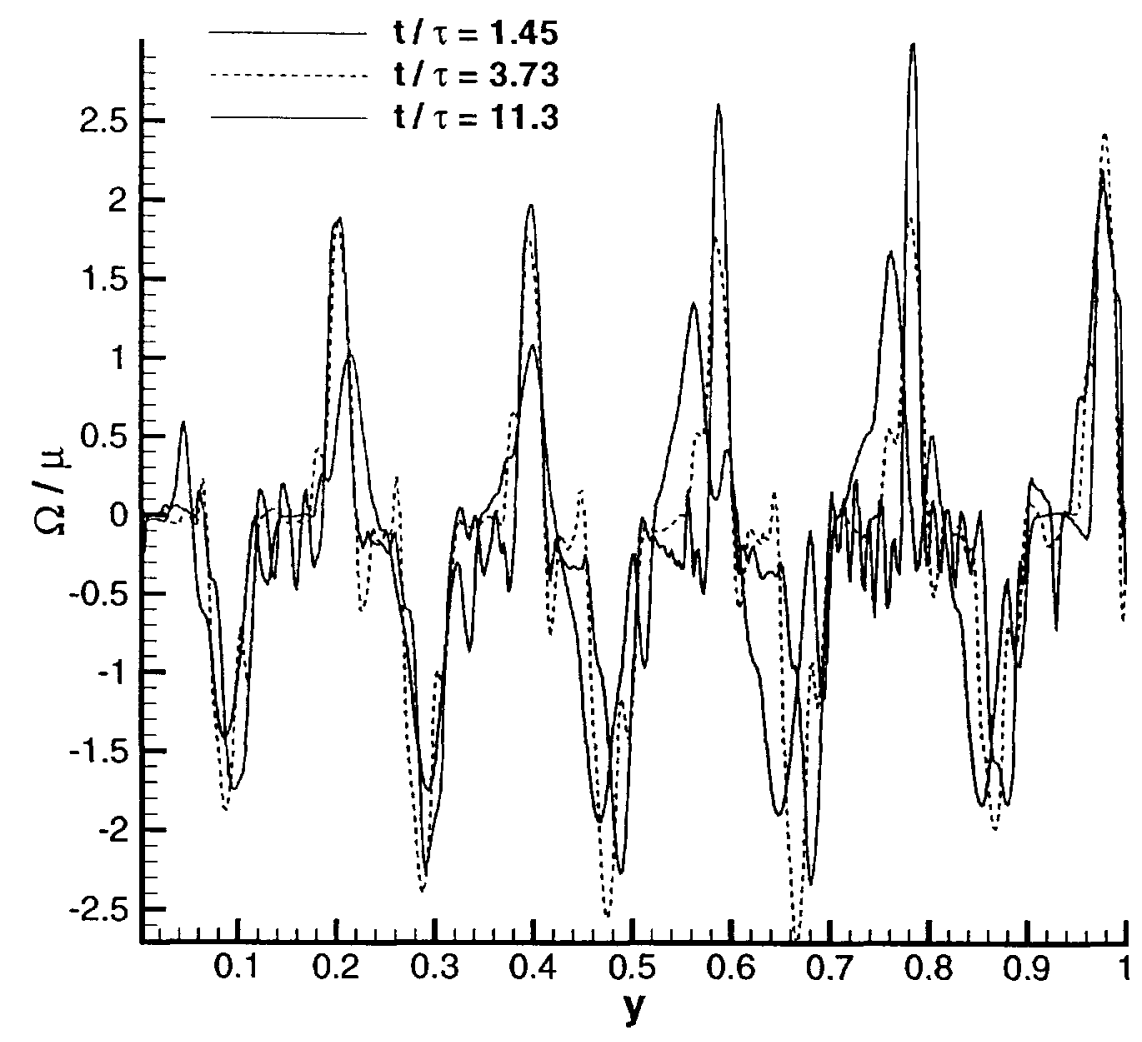

Figure 6: $\Omega / \mu$ for the $M=1.5, \eta=3.0$ Air-R22 interaction. We see that $\mu$ collapses the data between 0.5 and 2.0. We see that the negative peaks are consistently larger (in magnitude) than the positive one since the circulation on the sinusoidal perturbation is also negative and adds to the Haars. The early time "spiky" distribution evolves into a sawtooth one around $t / \tau \sim 4$ and persists till $t / \tau \sim 10$. The dotted, dashed and solid lines are for $t / \tau=1.45,3.73$ and 11.3 . 


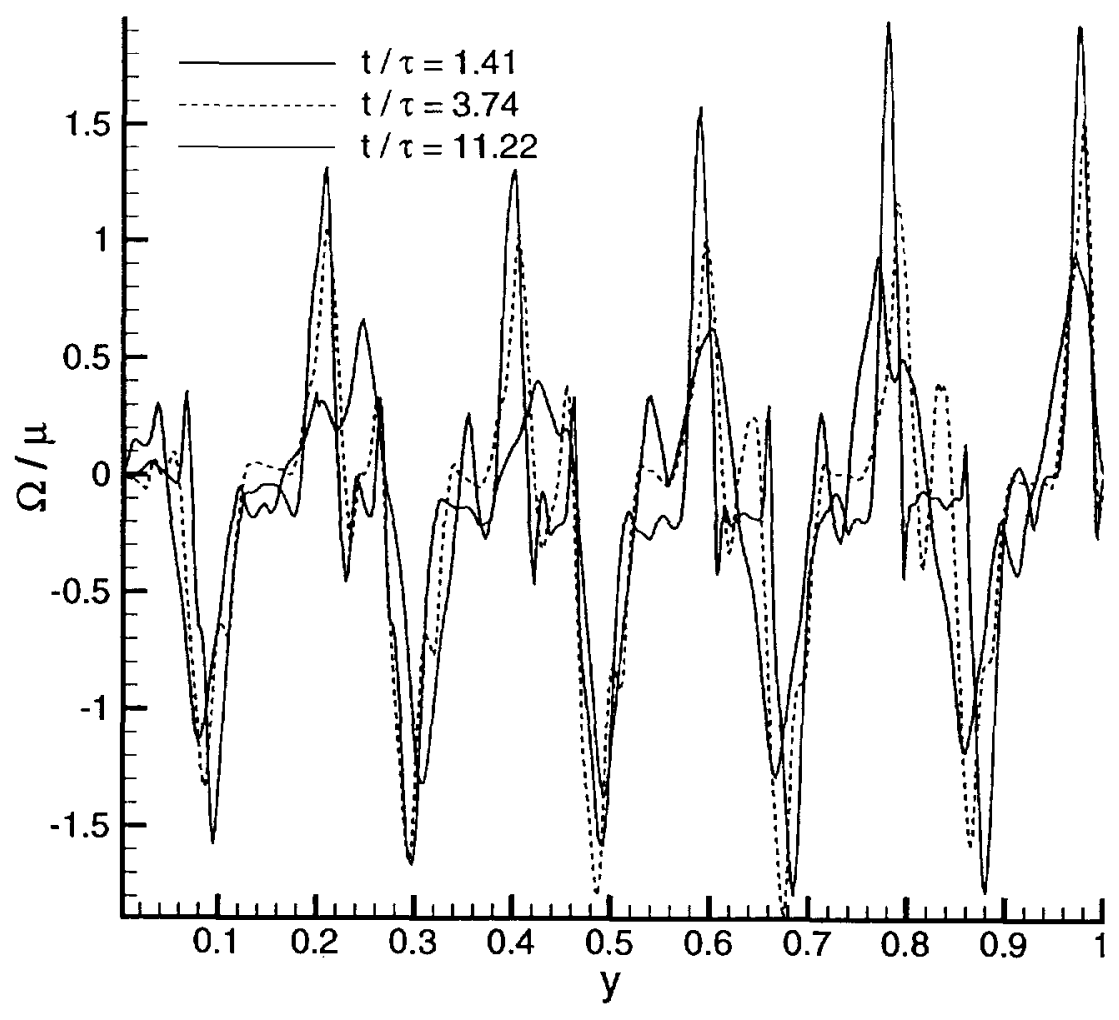

Figure 7: $\Omega / \mu$ for the $M=10, \eta=10.0$ interaction. Qualitatively, the results are the same as Fig. 6. However, the stronger interaction, with roughly 20 times more vorticity (see Fig. 5) causes the Haar-generated dipole growth to proceed faster. As seen in Fig. 4 , by $t / \tau=5.99$, the dipole widths are approximately $S_{j}$; by $t / \tau=12.04$, dipole-dipole interactions are very evident and strong. This is seen in the structure of the $\Omega$ plot; instead of a peak and a trough in a domain $S_{j}$, one sees multiple peaks/troughs corresponding to the partially-merged state of the dipoles (see Fig. 4(d)). The dotted, dashed and solid lines are for $t / \tau=1.41,3.74$ and 11.22 


\section{References}

[1] R.D. Richtmyer. Taylor instability in shock acceleration of compressible fluids. Comm. Pure and Appld. Math., XIII:297-319, 1960.

[2] Yu Meshkov. Instability of a shock wave accelerated interface between two gases. NASA Tech. Trans., NASA TT F-13074, 1970.

[3] J. Yang, T. Kubota, and E. E. Zukoski. Applications of shock induced-mixing to supersonic combustion. AIAA J., 31(5):854-862, 1993.

[4] D. L. Lindl, R. L. McCrory, and E. M. Campbell. Progress towards ignition and burn propagation in inertial confinement fusion. Physics Today, pages 32-40, September 1992.

[5] Philip A. Thompson. Compressible-Fluid Dynamics. McGraw-Hill, 1972.

[6] J.F. Hawley and N.J. Zabusky. Vortex paradigm for shock accelerated density stratified interfaces. Phys Rev Lett, 63:1241-1244, 1989.

[7] B. Sturtevant. Rayleigh-taylor instability in compressible fluids. In Proceedings 16th Int.Symp. on Shock Tubes and Waves, 1987.

[8] X. Yang, I-L. Chern, N. J. Zabusky, R. Samtaney, and J. F. Hawley. Vorticity generation and evolution in shock-accelerated density-stratified interfaces. Phys. Fluids A., 4:1531-1540, 1992.

[9] R. Samtaney and N.J. Zabusky. Circulation deposition on shock-accelerated planar and curved density stratified interfaces : Models and scaling laws. J. Fluid Mech., 269:45-85, 1994.

[10] R. Samtaney, J. Ray, and Norman J. Zabusky. Baroclinic circulation generation on shock accelerated slow/fast gas interfaces. Phys. Fluids, 10(5):1217-1230, May 1998.

[11] W. Mulder, S. Osher, and J.A: Sethian. Computing interface motion in compressible gas dynamics. J. Comp. Phys., 100:p209, 1992.

[12] B. Sturtevant. Rayleigh-taylor instabilities in compressible fluids. Technical report, 1985.

[13] O. Sadot, L. Erez, U. Alon, D. Oron, L. A. Levin, G. Erez, G. Ben-Dor, and D. Shvarts. Experimental and theoretical study of nonlinear evolution of single-mode and two-bubble interaction under richtmyer-meshkov instability. In Proceedings of the 6rd International Workshop on the Physics of Compressible Turbulent Mixing, 1997. 
[14] P. Collela. A direct eulerian muscl scheme for gas dynamics. SIAM J. Sci. Stat. Comput., 6:104-117, 1985.

[15] B. Van Leer. Towards the ultimate conservative scheme iv : A new approach to numerical convection. J. Comp. Physics, 23:276-299, 1977.

[16] J. Smoller. Shock Waves and Reaction-Diffusion Equations, Series of Comprehensive Studies in Mathematics. Springer-Verlag, 1982

[17] G. Strang. On the construction and comparision of difference schemes. SIAM J. Numer. Anal., 5:506517,1968 


\section{A Numerical method}

Our numerical method is a second-order accurate Godunov scheme and includes interface tracking. This method is similar to the MUSCL [14] scheme. We will present the method in 1D i.e. for $v=\mathcal{G}=0$. The governing equations for the flow are then

$$
\mathbf{U}_{\ell}+\mathcal{F}(\mathbf{U})_{x}=0
$$

By including the level set functions in the system of equations, the conservation laws are not strictly hyperbolic. In the non-conservative form, the equation is written as

$$
\mathbf{U}_{\imath}^{*}+A_{J} \mathbf{U}_{\boldsymbol{x}}^{*}=0
$$

where

$$
\mathrm{U}^{*}=\{\rho, u, p, \zeta\}^{T}
$$

and

$$
A_{J}=\frac{\partial \mathcal{F}}{\partial \mathbf{U}^{*}}
$$

$\zeta$ is used to determine the volume fraction of each gas in each cell and interpolate $\gamma$ linearly between $\gamma_{0}$ and $\gamma_{b}$. The eigenvalues $\lambda_{k}$ of $A$ are $u-c, u, u+c$ and $u$, where $c=(\gamma p / \rho)^{\frac{1}{2}}$ is the sound speed. The set of left eigenvectors corresponding to the eigenvalues above are the rows of the matrix $L$

$$
L=\left(\begin{array}{cccc}
0 & \rho / 2 & -1 /(2 c) & 0 \\
c & 0 & -1 / c & 0 \\
0 & \rho / 2 & 1 /(2 c) & 0 \\
0 & 0 & 0 & 1
\end{array}\right)
$$

We may uncouple the equations by multiplying both the terms by $L$ and use $\mathrm{V}, \mathrm{V}=L \mathrm{U}^{*}$, as the dependent variable.

The computational domain is divided into uniform cells of width $\Delta x$ and labeled $i=1 \ldots N$. The $i$-th cell has interfaces at $x_{i-1 / 2}$ and $x_{i+1 / 2}$, the left boundary being the $i$ th interface. Assuming that $\mathrm{U}_{i}^{n}$ $\left(\mathrm{U}\left(x_{i}, t^{n}\right)\right)$ is known, we calculate $\mathrm{U}_{i}^{n+1}$. We calculate $\mathrm{U}^{*}$ from $\mathrm{U}$, and denote this as $\overline{\mathrm{U}}^{*}$ as these are cell average values.

A piecewise linear distribution of $\mathbf{U}^{*}$ is constructed in each cell using $\overline{\mathbf{U}}^{*}$. To mitigate small wavelength oscillations Van Leer's monotonicity constraints [15] are applied in reconstructing the slope. Let $M_{i}$ 
represents the slope (actually the vector of slopes) in cell $i$. Then we have

$$
\begin{aligned}
& M_{i}=\min \left(0.5 L_{i}\left(\overline{\mathbf{U}}_{i+1}^{*}-\overline{\mathbf{U}}_{i-1}^{*}\right), 2.0 L_{i}\left(\overline{\mathbf{U}}_{i}^{*}-\overline{\mathbf{U}}_{i-1}^{*}\right), 2.0 L_{i}\left(\overline{\mathbf{U}}_{i+1}^{*}-\overline{\mathbf{U}}_{i}^{*}\right)\right) / \Delta x \\
& M_{i}=0, \quad \text { if } L_{i}\left(\overline{\mathbf{U}}_{i+1}^{*}-\overline{\mathbf{U}}_{i}^{*}\right) L_{i}\left(\overline{\mathbf{U}}_{i}^{*}-\overline{\mathbf{U}}_{i-1}^{*}\right)<0
\end{aligned}
$$

We now have a linear distribution of $V_{i}^{n}$ in each cell as

$$
\mathrm{V}_{i}^{n}=\overline{\mathbf{V}}_{i}^{n}+M_{i}\left(x-x_{i}\right)
$$

where $\overline{\mathbf{V}}_{i}^{n}=L_{i} \bar{U}_{i}^{*}$. We then solve the uncoupled equations at the cell interfaces at time $t=t_{n+1 / 2}$. This gives the value for the right side of the $\mathrm{i}$-th interface as

$$
\begin{aligned}
& V_{k, i, r}^{n+1 / 2}=\bar{V}_{k, i}^{n}-M_{k, i} / 2\left(\Delta x_{i}+\lambda_{k, i} \Delta t\right) \quad \text { if } \quad \lambda_{k, i}<0 . \\
& V_{k, i, r}^{n+1 / 2}=\bar{V}_{k, i}^{n} \quad \text { if } \quad \lambda_{k, i}>0 .
\end{aligned}
$$

The left state, $V_{k, i, l}^{n+1 / 2}$, can be obtained similarly.

$k=1,2,3,4$ are for the four elements in the $\mathrm{V}$ vector. We recover the values of $\mathbf{U}^{*}$ on the right and left sides of each cell interface as $U_{i, l}^{*}=L_{i-1}^{-1} V_{i, l}$ and $U_{i, r}^{*}=L_{i}^{-1} V_{i, r}$. Once we have the flow quantities at the interfaces at $t=t_{n+1 / 2}$ we solve the $1 \mathrm{D}$ Riemann problem, given a left and a right state $\mathrm{U}_{L}, \mathrm{U}_{R}$. Details of the Riemann problem are available in [16]. The interfacial value of $\zeta$ is $\zeta_{i, l}$ or $\zeta_{i, r}$ if the interface moves to the right or left respectively. We use $\zeta$ to calculate $\gamma$ at a given point and form the interface fluxes. These are then used to obtain the new solution according to

$$
\overline{\mathbf{U}}_{i}^{n+1}=\overline{\mathbf{U}}_{i}^{n}-\frac{\Delta t}{\Delta x_{i}}\left(\mathcal{F}\left(\mathbf{U}_{i+1 / 2}^{n+1 / 2}\right)-\mathcal{F}\left(\mathbf{U}_{i-1 / 2}^{n+1 / 2}\right)\right) .
$$

$\Delta t$ is calculated using a CFL less than half. This method is extended to multiple dimensions easily. For $2 \mathrm{D}$, alternating $\mathrm{x}$ and $\mathrm{y}$ sweeps generate second order accuracy formally [17]. 


\section{B Projection of Wavelets onto a Spherical Harmonic Basis}

As mentioned previously, wavelet analysis and Fourier analysis provide different and complementary ways to represent information. The basis vectors for Fourier analysis represent global information. Wavelets, on the other hand, are completely local in nature. The relative efficiency of these two basis sets will simply depend on the nature of the information being represented. If the data is completely local in nature, then one would find that wavelet analysis provides a very efficient basis set. And, if the data is global in nature then Fourier analysis will be the preferred basis. There are, however, many situations in physics where the data is primarily composed of large scale slowly varying features with localized small-scale phenomenon appearing randomly. One can think of the large-scale basis functions in Fourier analysis as representing the global periodic large-scale features. Then one would superimpose upon this the small scale locally varying features using wavelet basis vectors. If, per chance, the small scale information is global then one would opt to use the small-scale eigenvectors from Fourier analysis. Precisely speaking one must look at the decay rates of the expansions in both the Fourier basis vectors and the wavelet basis vectors. Further, one must look at the projection of the wavelet basis functions onto the Fourier basis vectors. This information is sufficient to form an effective combination of Fourier analysis and wavelet analysis.

For Fourier analysis we will have a representation of the form,

$$
D=\sum_{j} \lambda_{j} V_{j} V_{j}^{H}
$$

where $\lambda_{j}$ is the coefficient for the $j-t h$ basis function $V_{j}$. As mention above, we assume the basis vectors are ordered from largest to smallest, i.e., $\lambda_{j}>\lambda_{j+1}$. Ordered in this manner we have a decaying spectrum.

On the other hand, we can represent the data in wavelet basis with the notation,

$$
D=\sum_{j, k} d_{j, k} W_{j, k}
$$

We are assuming that the data $D$ is finite and thus our two representations are complete and the representations as written above are lossless.

Let us consider a slightly more general scenario with simplified notation and in a continuous domain. If we have two different orthonormal bases sets $B_{1}$ and $B_{2}$ with,

$$
P_{B^{1}} f(x)=\sum_{m} a_{m}^{1} B_{m}^{1}(x)
$$

with

$$
a_{m}^{1}=\int f(x) B_{m}^{1}(x) d x
$$


and the second representation in terms of the second basis,

$$
P_{B^{2}} f(x)=\sum_{n} a_{n}^{2} B_{n}^{2}(x)
$$

with

$$
a_{n}^{2}=\int f(x) B_{n}^{2}(x) d x
$$

then if one projects the representation in one basis set onto the second basis set, one obtains,

$$
P_{B^{1}} P_{B^{2}} f(x)=\sum_{m} a_{m}^{1} B_{m}^{1}(x)
$$

where,

$$
a_{m}^{1}=\int\left(\sum_{n} a_{n}^{2} B_{n}^{2}(x)\right) B_{m}^{1}(x) d x
$$

or,

$$
a_{m}^{1}=\sum_{n} a_{n}^{2} \int B_{n}^{2}(x) B_{m}^{1}(x) d x
$$

so that the key to mapping from one basis set to the other basis is the matrix $M_{n, m}=<B_{n}^{2}, B_{m}^{1}>$. For the projection of wavelets onto Fourier modes we would, therefore, be interested in the behavior of,

$$
p_{j, k, n}^{\text {continuous }}=\int W_{j, k}(x) V_{n}(x) d x
$$

written in a continuous variable or

$$
p_{j, k, n}^{\text {discrete }}=W_{j, k}^{T} V_{n}
$$

in discrete notation. In either case, the number $p_{j, k, n}$ is a measure of the similarity of the two basis vectors. So that as one projects wavelet basis functions of increasing scale onto Fourier basis functions of decreasing scale, one expects the projection to decrease in amplitude. Further, one expects that there will be a wavelet scale which most closely matches the predominant scale in a given Fourier basis vector.

\section{B.1 Combining Spherical Harmonics and Wavelet Analysis}

As mentioned above, our overall goal in this manuscript is to combine the strong features of Fourier analysis with the strong features of wavelet analysis. We envision a function or data composed of contributions from both basis sets. For now we will not write limits on the integrals and summations. Such limits will be chosen so that various error criteria are satisfied, as outlined below. So, our expansion will be of the form,

$$
f(x)=\sum_{i} \lambda_{i} V_{i}(x)+\sum_{j, k} d_{j, k} W_{j, k}(x)
$$


Since the Fourier basis vectors are not orthogonal to the wavelets, the above representation has some redundancy or error that is proportional to the projection of the wavelets onto the Fourier modes, as explained above. That is,

$$
d_{j, k}=\int f(x) W_{j, k}(x) d x-\sum_{i} \lambda_{i} \int V_{i}(x) W_{j, k}(x) d x
$$

where the term

$$
E_{j, k}^{a}=\sum_{i} \lambda_{i} \int V_{i}(x) W_{j, k}(x) d x,
$$

represents the error in the above inner product of the wavelet with the surface data. This error, as discussed above, can be made as small as one desires by appropriately choosing which wavelets and Fourier modes to use in the expansion and by considering the spectrum in both the wavelet and Fourier basis sets.

Conversely, the Fourier coefficient represents the projection of the data onto the corresponding Fourier basis vector and there will be a similar error term which depends ultimately on the projection of the wavelet basis functions on the Fourier basis vectors. That is,

$$
\lambda_{i}=\int f(x) V_{i}(x) d x-\sum_{j, k} d_{j, k} \int W_{j, k}(x) V_{i}(x) d x
$$

and the error term is,

$$
E_{i}^{b}=\sum_{j, k} d_{j, k} \int W_{j, k}(x) V_{i}(x) d x
$$

So, if we define $\epsilon$ to be,

$$
\epsilon_{i, j, k}=\int W_{j, k} V_{i} d x
$$

then we can see that our two errors are

$$
E_{j, k}^{a}=\sum_{i} \lambda_{i} \epsilon_{i, j, k}
$$

and,

$$
E_{i}^{b}=\sum_{j, k} d_{j, k} \epsilon_{i, j, k}
$$

Therefore, we can have very accurate estimates of the errors introduced by combining the two basis sets simply by using the above two expressions which require that we know the Fourier spectrum $\lambda_{i}$, the wavelet spectrum $d_{j, k}$ and the projection of the basis functions onto each other $\epsilon_{i, j, k}$.

The projection of wavelets onto Fourier modes or Fourier modes onto wavelets can be viewed also as the interference between the two representations. In principle we would prefer that this inference be zero, but it will not be due to the lack of orthogonality. Whereas wavelets have the ability detect scales with respect to position, the Fourier basis functions do not. We, therefore, need to modify our $\epsilon_{i, j, k}$ so that the position 
parameter $k$ is eliminated. We can do this in a number of ways, but we keep the presentation in the $L_{2}$ norm and simply sum up the square of the interactions over $k$,

$$
\bar{\epsilon}_{i, j}=\sum_{k}\left(\epsilon_{i, j, k}\right)^{2}
$$

and in this manner we obtain a measure of the interaction or interference of the Fourier mode $V_{i}$ with the wavelets at wavelet scale $j$.

¿From the above section on wavelet analysis, we recall that we denote the finest wavelet scale of information as $j=0$ and more coarse scales as $j$ increases. Further, we denote the subspace spanned by wavelets at scale $j$ as $W_{j}$. So, our $\bar{\epsilon}_{i, j}$ is a measure of the orthogonality of the subspace $W_{j}$ with the subspace spanned by the eigenvector $V_{i}$. Though the issue of scale size for eigenvector $V_{i}$ is not well defined, we do expect for physical reasons that scales of information in $V_{i}$ will be larger than the scales of information in $V_{i+1}$. We expect this due to the previously defined ordering of the eigenvectors. That is, we expect that the overall general flow of the ocean model will have most of its energy in the larger scales with the smaller scales being less and less energetic as the scale size gets smaller. And, since the eigenvalues represent the energy in a given eigenvector we expect that the larger eigenvalues will correspond to larger scales.

\section{B.2 Role of Different Wavelets}

As mentioned above, we limit our study to the family of orthogonal wavelets known as the Daubechies wavelets. So, within this family of wavelets which one should we choose to work with. This will depend on a few factors

First of all, as one goes to higher and higher order wavelets, one finds that the non-zero region of the wavelet grows. That is the compact support of the wavelets is certainly maintained, but the support grows. Further, when the wavelets become higher and higher order, there is a region of their support which is nonzero but which can be very close to zero. Generally this close-to-zero area of support is not a desirable property and should generally be avoided in computer computations where roundoff error can become an issue for quantities close to zero

A second issue is the overall magnitude of the projection of the wavelets onto the Fourier modes. From the approximation theory presented above, one can see that in the wavelet approximation of sine waves that the truncation error, hence projection size, is governed by traditional polynomial truncation error. So, one expects an expression of the form

$$
\operatorname{Proj}=\frac{\left(2^{j} \Delta x\right)^{p+1} k^{p+1}}{(p+1) !}
$$


parameter $k$ is eliminated. We can do this in a number of ways, but we keep the presentation in the $L_{2}$ norm and simply sum up the square of the interactions over $k$,

$$
\bar{\epsilon}_{i, j}=\sum_{k}\left(\epsilon_{i, j, k}\right)^{2}
$$

and in this manner we obtain a measure of the interaction or interference of the Fourier mode $V_{i}$ with the wavelets at wavelet scale $j$.

¿From the above section on wavelet analysis, we recall that we denote the finest wavelet scale of information as $j=0$ and more coarse scales as $j$ increases. Further, we denote the subspace spanned by wavelets at scale $j$ as $W_{j}$. So, our $\bar{\epsilon}_{i, j}$ is a measure of the orthogonality of the subspace $W_{j}$ with the subspace spanned by the eigenvector $V_{i}$. Though the issue of scale size for eigenvector $V_{i}$ is not well defined, we do expect for physical reasons that scales of information in $V_{i}$ will be larger than the scales of information in $V_{i+1}$. We expect this due to the previously defined ordering of the eigenvectors. That is, we expect that the overall general flow of the ocean model will have most of its energy in the larger scales with the smaller scales being less and less energetic as the scale size gets smaller. And, since the eigenvalues represent the energy in a given eigenvector we expect that the larger eigenvalues will correspond to larger scales.

\section{B.2 Role of Different Wavelets}

As mentioned above, we limit our study to the family of orthogonal wavelets known as the Daubechies wavelets. So, within this family of wavelets which one should we choose to work with. This will depend on a few factors.

First of all, as one goes to higher and higher order wavelets, one finds that the non-zero region of the wavelet grows. That is the compact support of the wavelets is certainly maintained, but the support grows. Further, when the wavelets become higher and higher order, there is a region of their support which is nonzero but which can be very close to zero. Generally this close-to-zero area of support is not a desirable property and should generally be avoided in computer computations where roundoff error can become an issue for quantities close to zero.

A second issue is the overall magnitude of the projection of the wavelets onto the Fourier modes. From the approximation theory presented above, one can see that in the wavelet approximation of sine waves that the truncation error, hence projection size, is governed by traditional polynomial truncation error. So, one expects an expression of the form,

$$
\operatorname{Proj}=\frac{\left(2^{j} \Delta x\right)^{p+1} k^{p+1}}{(p+1) !}
$$


where $p$ is the number of vanishing moments of the wavelet and $k$ is the $k-t h$ sine mode being approximated. From this expression one can see that as $p$ increases that the magnitude of the projection and hence interference between the wavelet basis vectors and the Fourier modes will decrease

As indicated above, an upper bound on the size of projection of a wavelet onto a sine wave can be found from truncation error analysis of traditional polynomial interpolation. Therefore, if our eigenvector is now set to be a $\sin$ wave, $V_{n}=e^{i n x}$, then our projection,

$$
\epsilon_{n, j, k}^{p}=\int W_{j, k}(x) e^{i n x} d x
$$

will have an upper bound of,

$$
\max _{k}\left|\epsilon_{n, j, k}^{p}\right| \leq \frac{\left(2^{j} \Delta x\right)^{p+1} n^{p+1}}{(p+1) !},
$$

where $p$ is the number of vanishing moments of the wavelet and $\Delta x$ is the distance between grid points in the original data sample. Since this expression is an upper bound, it should only be used as a rough guide to the projection magnitude.

Now that we have a precise expression for $\epsilon$, we can assume a form for the Fourier spectrum and thereby obtain a more precise estimate of the error in mixing a Fourier basis with a wavelet basis. If we assume a Fourier spectrum of the form

$$
\lambda_{n}=n^{-m} e^{a * \frac{n}{n_{0}}},
$$

then we can see that error $E_{j, k}^{a}$ as defined above will be bounded above by

$$
E_{j, k}^{a} \leq\left|\sum_{n} \frac{n^{p-n+1}}{(p+1) !}\left(2^{j} \Delta x\right)^{p+1} e^{a * \frac{n}{n_{0}}}\right| \leq \sum_{n}\left|\frac{n^{p-n+1}}{(p+1) !}\left(2^{j} \Delta x\right)^{p+1} e^{a * \frac{n}{n_{0}}}\right| .
$$

This upper bound on error can be used to estimate the interference between the wavelet and the Fourier basis vectors and in the context of surface structure estimation, this interference can be made as small as is desired by choosing which Fourier modes and which wavelet scales to use. 